\title{
ORIGINAL
}

\section{Benign cystic nodules may have ultrasonographic features mimicking papillary thyroid carcinoma during interval changes}

\author{
Sang Soo Kim ${ }^{1)}$, 3), Mi Ra Kim¹), Ji Young Mok $^{1)}$, Jung Eun Huh ${ }^{1)}$, Yun Kyung Jeon ${ }^{1)}$, Bo Hyun Kim ${ }^{1)}$, \\ Seong-Jang $\mathrm{Kim}^{3)}$, Yong Ki Kim${ }^{2}$ and $\mathrm{In}$ Joo Kim ${ }^{1)}$, 3) \\ 1) Department of Internal Medicine, Pusan National University Hospital, Busan, Korea \\ ${ }^{2)}$ Kim Yong Ki Internal Medicine Clinic, Busan, Korea \\ ${ }^{3)}$ Medical Research Insititute, Pusan National University, Busan, Korea
}

\begin{abstract}
It had been observed that some cystic nodules change morphologically with ultrasonographic (US) features suspicious for malignancy. The aim of this study was to evaluate the US characteristics of benign cystic nodules mimicking papillary thyroid carcinoma (PTC) during interval changes. Between January 2009 and October 2009, 26 patients with benign cystic nodules showing marked hypoechogenicity in US during the follow-up period were enrolled. During the same period, 38 patients with marked hypoechogenicity in US were enrolled for the PTC group. We evaluated the differences in US characteristics between the 2 groups. Nodule size, margin, echogenic dot and vascularity were not significantly different between the 2 groups. Nodule shape was significantly different between the 2 groups with a lower prevalence of taller than wide in the benign cystic group $(11.5 \%$ vs. $39.5 \%, P=0.022)$. Other coexisting cystic nodules were more frequently observed in benign cystic group (48.3\% vs. 5.3\%, $P=0.001)$. If echogenic dot was detected in benign cystic nodule, it was more than $1 \mathrm{~mm}$ in size without posterior acoustic shadowing unlike echogenic dots in the PTC group. In conclusion, some of the benign cystic nodules may have suspicious malignant features on US during interval changes. A careful assessment of US findings and a previous history may be of value in discriminating them from PTC.
\end{abstract}

Key words: Colloid cyst, Ultrasonography, Papillary carcinoma, Thyroid nodule

\begin{abstract}
ALTHOUGH the incidence of thyroid cancers may be correlated with the development of improved diagnostic and testing technologies, it is increasing rapidly [1]. Thyroidultrasonography(US) is a widespread technique that is used as a first-line diagnostic procedure in thyroid disease, several studies have been performed to establish its ability to differentiate between benign and malignant lesions [2-4]. Certain US features of thyroid nodules can be helpful in identifying nodules most at risk for malignancy including hypoechogenicity, increased vascularity, microcalcifications, irregular margins and the absence of a halo [5]. When a thyroid nodule is "markedly hypoechoic," as being much less echogenic

Received Mar. 22, 2011; Accepted May 3, 2011 as K11E-095 Released online in J-STAGE as advance publication May 26, 2011

Correspondence to: In Joo Kim, M.D., Ph.D., Division of Endocrinology and Metabolism, Department of Internal Medicine, Pusan National University Hospital, 305 Gudeok-Ro, Seo-Gu, Busan 602-739, Korea. E-mail: injkim@pusan.ac.kr
\end{abstract}

than the medium-level echogenicity of the strap muscles, the specificity for detection of malignancy is increased to $94 \%$, although the sensitivity is reduced [6]. Thus, markedly hypoechogenicity in thyroid nodules is highly suggestive US feature for papillary thyroid carcinoma (PTC).

It had been observed that some cystic nodules change morphologically with marked hypoechogenicity on US, becoming difficult to distinguish from PTC, during follow-ups in clinical practice (Fig. 1). The aim of this study was to evaluate the clinical implications of the change of benign cystic nodules to malignancymimicking features in US and to determine US characteristics differentiating benign cystic nodule with malignancy-mimicking features from PTC.

\section{Patients and Methods}

Between January 2009 and October 2009, 1,326 


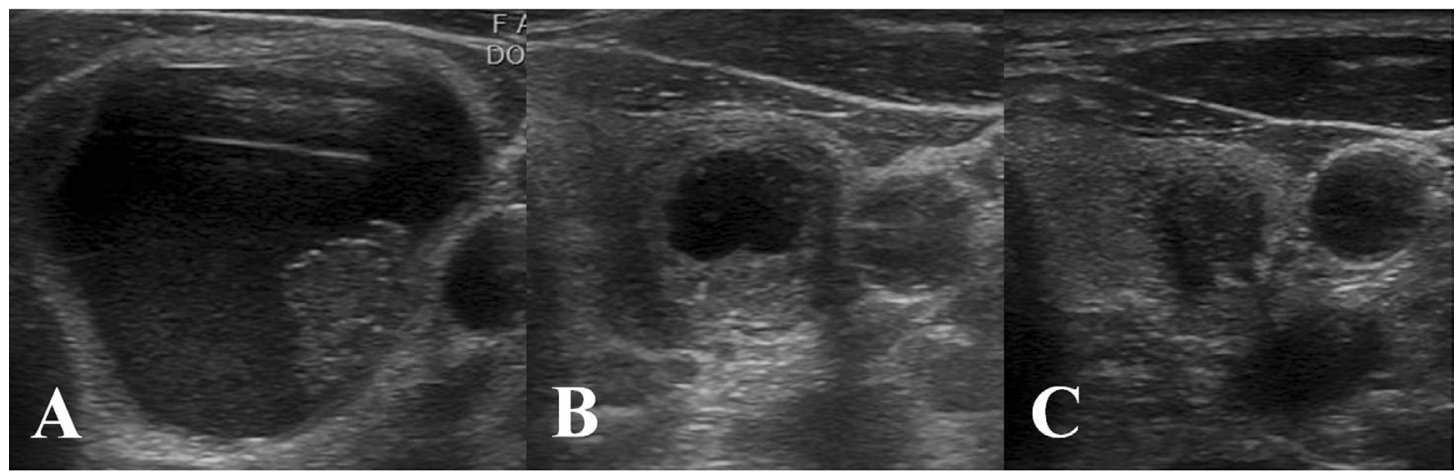

Fig. 1 (A) Case 25. A 47-year-old woman who were diagnosed with benign cystic nodule twice by fine needle aspiration cytology during the follow-up 13 months ago. Transverse sonogram shows a 3.5-cm predominantly cystic nodule. (B) The same nodule shrank about 50\% in size after colloid aspiration 6 month later. Transverse sonogram shows 1.7 -cm predominantly cystic nodule. (C) The US nature of the same nodule was changed 7 month later. Transverse sonogram shows a 0.8 -cm nodule with taller than wide shape, ill-defined margin and marked hypoechogenicity.

patients visited our thyroid clinic in Pusan National University Hospital for follow-ups of known benign thyroid nodules. Of the 1,326 patients, 26 met the following criteria (Benign cystic group): (1) purely or predominantly cystic nodule on a previous US; excluded cystic nodule possessing a solid component with vascularity, solid excrescences or microcalcification, (2) markedly hypoechoic echogenicity on US during follow-up periods and (3) benign cytologic confirmation more than twice. Patients with a previous history of sclerotherapy with ethanol were excluded. During the same period, 262 consecutive patients underwent thyroid surgery for PTC at our hospital. Because cystic benign nodules with small size (all of them, $<1 \mathrm{~cm}$ ) have ambiguous features in discrimination against PTC on US, 38 patients with PTC were enrolled as PTC group according to the following criteria: (1) markedly hypoechoic echogenicity on US, (2) tumor $<1 \mathrm{~cm}$ on US and (3) pathologic confirmation of PTC.

US was performed with a US scanner (HDI 5000, Philips Medical Systems, Bothell, WA and Vivid $i$, GE Healthcare, Milwaukee, WI) equipped with a $10-13 \mathrm{MHz}$ linear probe. The US examination was performed by 2 endocrinologists (S. S. Kim, Y. K. Jeon) according to their schedule during this study period. Three endocrinologists (S. S. Kim, Y. K. Jeon, B. H. Kim) reviewed the US imaging blinded to the final diagnosis. The US characteristics we used in this study were based on previously defined criteria for discriminating malignant from benign nodules [6]. A markedly hypoechoic echogenicity was defined as a decreased echogenicity compared with the surrounding strap muscles. A nodule with a taller than wide shape was defined as being greater in the anteroposterior dimension than the transverse dimension. A purely cystic and a predominantly cystic nodule were defined as possessing a cystic component of $>90 \%$ and $>50 \%$, respectively.

Informed consent was obtained from each participant at the time of surgery or fine-needle aspiration. This retrospective review protocol was approved by the Institutional Review Board at Pusan National University Hospital.

\section{Statistical analysis}

All statistical analyses were performed with SPSS version 12.0 (SPSS, Chicago, IL). The statistical difference between groups was assessed using the Mann-Whitney $U$ test. The $\chi^{2}$ test or Fisher exact test was performed to analyze categorical data as appropriate. A $p$ value of $<0.05$ was considered statistically significant.

\section{Results}

Twenty-six patients (22 women, 4 men; age range, 17-77 years; median age, 53.5 years) were included in this study (Table 1). The median interval between initial diagnosis of cystic nodule and the appearance of suspicious US features was 15.4 months (range, 5.793.7 months). Of 26 patients in the benign cystic group, 17 (65.4\%) initially represented a predominantly cystic nature in US.

As shown in Table 2, nodule size, margin, echogenic 
Table 1 Characteristics of the Benign Cystic Group

\begin{tabular}{|c|c|c|c|c|c|c|c|}
\hline $\begin{array}{l}\text { Patient } \\
\text { No. }\end{array}$ & Sex & $\begin{array}{l}\text { Age, } \\
\text { year }\end{array}$ & $\begin{array}{l}\text { Previous US } \\
\text { size, } \mathrm{cm}\end{array}$ & $\begin{array}{c}\text { Previous US } \\
\text { nature }\end{array}$ & $\begin{array}{c}\text { Follow-up } \\
\text { period, month }\end{array}$ & $\begin{array}{l}\text { Aspiration } \\
\text { of colloid }\end{array}$ & $\begin{array}{c}\text { LT4 } \\
\text { Suppression }\end{array}$ \\
\hline 1 & F & 51 & 2.60 & Purely cystic & 60 & Yes & No \\
\hline 2 & M & 49 & 1.46 & Purely cystic & 45 & No & Yes \\
\hline 3 & $\mathrm{~F}$ & 59 & 1.74 & Predominantly cystic & 94 & Yes & No \\
\hline 4 & $\mathrm{~F}$ & 48 & 0.98 & Purely cystic & 15 & No & No \\
\hline 5 & $\mathrm{~F}$ & 54 & 3.00 & Predominantly cystic & 51 & Yes & Yes \\
\hline 6 & $\mathrm{~F}$ & 57 & 2.04 & Predominantly cystic & 63 & No & Yes \\
\hline 7 & $\mathrm{~F}$ & 57 & 3.48 & Predominantly cystic & 19 & Yes & Yes \\
\hline 8 & M & 83 & 1.28 & Purely cystic & 15 & No & No \\
\hline 9 & $\mathrm{~F}$ & 59 & 2.04 & Predominantly cystic & 13 & No & Yes \\
\hline 10 & $\mathrm{~F}$ & 72 & 2.46 & Predominantly cystic & 17 & No & Yes \\
\hline 11 & F & 49 & 1.73 & Predominantly cystic & 16 & No & Yes \\
\hline 12 & $\mathrm{~F}$ & 55 & 4.48 & Predominantly cystic & 27 & Yes & Yes \\
\hline 13 & $\mathrm{~F}$ & 53 & 4.18 & Purely cystic & 11 & Yes & No \\
\hline 14 & $\mathrm{~F}$ & 21 & 2.53 & Predominantly cystic & 6 & Yes & No \\
\hline 15 & $\mathrm{~F}$ & 45 & 3.03 & Purely cystic & 24 & Yes & Yes \\
\hline 16 & $\mathrm{~F}$ & 25 & 2.56 & Purely cystic & 12 & Yes & No \\
\hline 17 & F & 22 & 2.08 & Purely cystic & 12 & Yes & No \\
\hline 18 & F & 58 & 2.50 & Predominantly cystic & 12 & Yes & No \\
\hline 19 & $\mathrm{~F}$ & 46 & 2.39 & Predominantly cystic & 7 & Yes & Yes \\
\hline 20 & M & 54 & 1.52 & Predominantly cystic & 14 & No & No \\
\hline 21 & $\mathrm{~F}$ & 60 & 3.89 & Purely cystic & 15 & Yes & No \\
\hline 22 & $\mathrm{~F}$ & 73 & 2.52 & Predominantly cystic & 13 & Yes & No \\
\hline 23 & $\mathrm{~F}$ & 41 & 1.92 & Predominantly cystic & 19 & Yes & Yes \\
\hline 24 & M & 55 & 2.07 & Predominantly cystic & 19 & Yes & No \\
\hline 25 & $\mathrm{~F}$ & 47 & 3.46 & Predominantly cystic & 13 & Yes & No \\
\hline 26 & $\mathrm{~F}$ & 42 & 1.86 & Predominantly cystic & 7 & Yes & No \\
\hline
\end{tabular}

US, ultrasonographic; LT4, levothyroxine;

Table 2 Ultrasonographic Characteristics Between the Benign Cystic and PTC Groups

\begin{tabular}{|c|c|c|c|c|}
\hline Parameters & Characteristics & $\begin{array}{c}\text { Benign Cystic } \\
\text { Group } \\
(\mathrm{n}=26)\end{array}$ & $\begin{array}{c}\text { PTC } \\
\text { Group } \\
(\mathrm{n}=38)\end{array}$ & $P$ value \\
\hline Size, cm & & $0.73 \pm 0.26$ & $0.68 \pm 0.20$ & 0.738 \\
\hline \multirow[t]{3}{*}{ Shape } & Ovoid to round & $13(50.0)$ & $5(13.2)$ & \multirow{3}{*}{0.002} \\
\hline & Irregular & $10(38.5)$ & $18(47.4)$ & \\
\hline & Taller than wide & 3 (11.5) & 15 (39.5) & \\
\hline Margin & Well-defined & $2(7.7)$ & $2(5.3)$ & \multirow{2}{*}{1.000} \\
\hline & Ill-defined & $24(92.3)$ & 36 (94.7) & \\
\hline Echogenic dot & Absence & 19 (73.1) & $32(84.2)$ & 0.277 \\
\hline Vascularity & Absence & $25(96.2)$ & $36(94.7)$ & 1.000 \\
\hline Coexisting nodule(s) & Absence & $6(23.1)$ & $12(31.6)$ & 0.457 \\
\hline Coexisting cystic nodule(s) & Absence & $11(42.3)$ & $2(5.3)$ & 0.001 \\
\hline
\end{tabular}

Data are expressed as mean \pm SD for continuous variables and frequency (\%) for categorical variables.

dot and vascularity were not significantly different between the 2 groups. The nodule shape was significantly different between the 2 groups $(P=0.002)$ with a lower prevalence of taller than wide shape in the benign cystic group ( $11.5 \%$ vs. $39.5 \%, P=0.022$ ) (Fig. 2 ). The sensitivity for predicting the papillary thyroid carcinoma in cases with taller than wide shape were
$39.5 \%$ and the specificity was $88.5 \%$. Of 7 patients with internal echogenic dot in the benign cystic group, all had echogenic dots $>1 \mathrm{~mm}$ without posterior acoustic shadowing (Fig. 3A). However, all of the 6 patients with echogenic dots in the PTC group had echogenic dots < $1 \mathrm{~mm}$ without posterior acoustic shadowing (Fig. 3B). As an additional US finding, there was no significant 


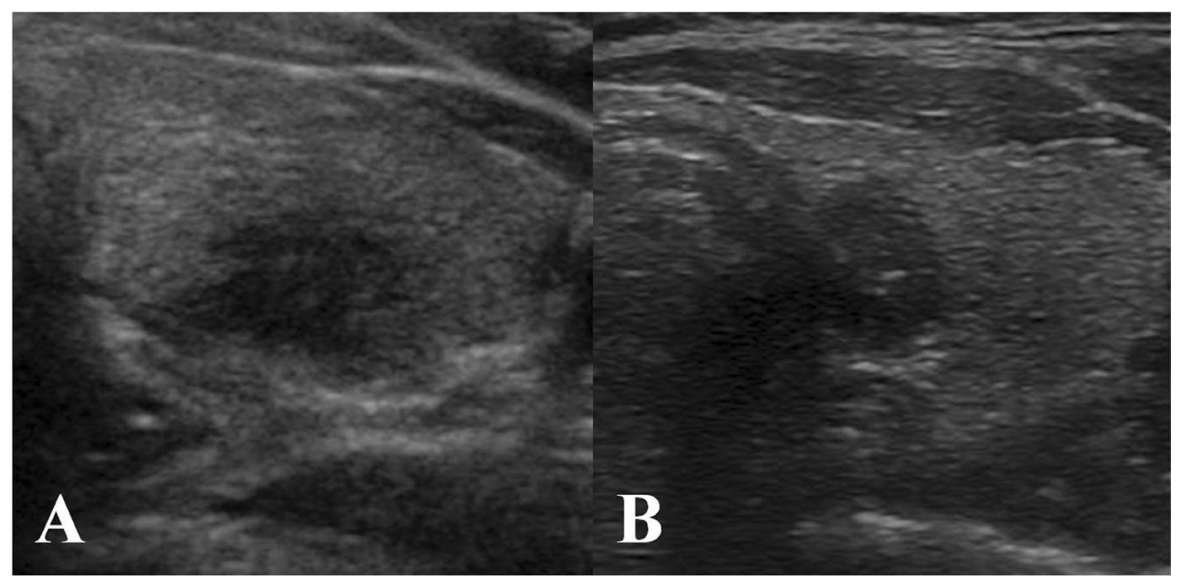

Fig. 2 (A) Case 11. A 49-year-old woman diagnosed with benign cystic nodule twice by fine needle aspiration cytology during the follow-up 16 months ago. Transverse sonogram shows $0.8-\mathrm{cm}$ nodule with irregular shape, ill-defined margin and marked hypoechogenicity. (B) A 47-year-old woman with PTC, diagnosed by surgical pathology. Transverse sonogram shows 0.8-cm nodule with taller than wide shape, ill-defined margin and marked hypoechogenicity.

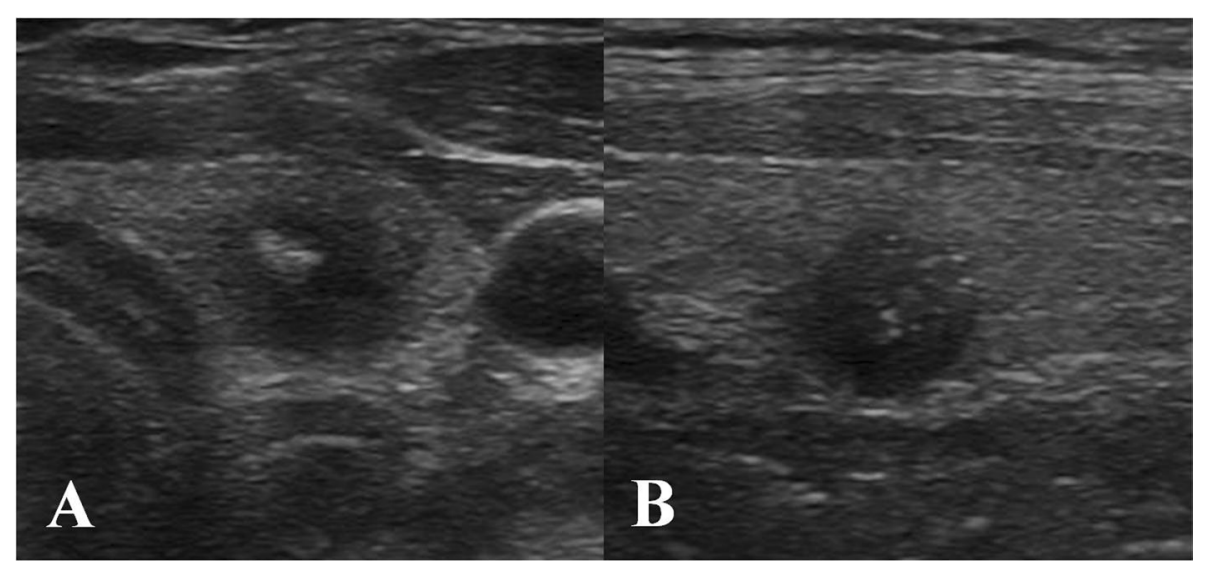

Fig. 3 (A) Case 12. A 55-year-old woman diagnosed with benign cystic nodule twice by fine needle aspiration cytology during the follow-up 27 months ago. Transverse sonogram shows a $0.9-\mathrm{cm}$ nodule with irregular shape, ill-defined margin, marked hypoechogenicity and internal echogenic dot without posterior acoustic shadowing. (B) A 48-year-old woman with PTC, diagnosed by surgical pathology. Longitudinal sonogram shows a $0.8-\mathrm{cm}$ nodule with round shape, well-defined margin, marked hypoechogenicity and internal echogenic spots without posterior acoustic shadowing.

difference between the 2 groups in coexisting thyroid nodule(s). However, other coexisting cystic nodules were more frequently observed in benign cystic group (48.3\% vs. $5.3 \%, P=0.001)$.

In cystic benign group, aspiration of the colloid component was performed on 18 patients (69.2\%), and levothyroxine was prescribed to 11 patients (42.3\%) for suppressing the nodule. The change of size in cystic nodules was greater after aspiration of the colloid than spontaneous observation $(2.0 \pm 0.7$ vs. $1.1 \pm 0.4$ $\mathrm{cm}, P=0.001)$. Ill-defined margin, taller than wide shape, echogenic dot and intranodular blood flow were found $100 \%, 12.5 \%, 12.5 \%$ and $0 \%$ of spontaneous observation group $(n=8)$, respectively. There were no significant differences in the US characteristics such as shape, margin, echogenic dot and vascularity depending on aspiration of the colloid component during follow-up of cystic benign nodule. In addition, the US characteristics of only spontaneous observation group were not significant different from those of PTC group. The US features of cystic nodules during follow-up were not significantly different between patients with and without levothyroxine suppression therapy. 


\section{Discussion}

The results of this study showed that some cystic nodules with morphological changes had US findings similar to PTC including marked hypoechogenicity and ill-defined margins during follow-ups. However, a nodule that was taller than wide was less frequently observed in the benign cystic group. In addition, internal echogenic dot $>1 \mathrm{~mm}$ without posterior acoustic shadowing and the presence of other coexisting cystic nodule may give us a clue in discriminating benign cystic tumors from PTC. Malignancymimicking features in benign cystic thyroid nodules with follow-up US after percutaneous ethanol injection or colloid aspiration have been reported [7, 8]. About $80 \%$ of the benign cystic nodules decreased in size or disappeared when they were examined 9 to 11 years later [9]. It has not been reported whether benign cystic nodules have malignancy-mimicking features in US after simple percutaneous aspiration or during the natural course.

Recently, a previous study was reported that benign cystic nodules after aspiration could have suspicious malignant features [8]. They reported that shadowing and a halo is only characteristic finding of benign cystic nodule. The previous study, which is insisting the resembling meaning on US examination with our study, had some different study design and results from our study. In our study, only nodules with marked hypoechogenicity were enrolled as our study cohort, which is main feature mimicking PTC on US. In addition, we also observed that some benign cystic nodules showed ambiguous features during their natural course without aspiration. For these reasons, it could be explained the discrepancy between 2 studies in US characteristics regarding to discriminating the benign cystic nodule from PTC.

Many studies have shown hypoechogenicity to be a finding suggestive of malignancy [10]. Hypoechogenicity in US is thought to result from increased cellularity and cellular compaction seen in PTC. Marked hypoechogenicity is strongly suggestive of malignancy [6], and this nature was also observed in benign cystic nodules in this study from its shrinkage. This feature occurred not only from the change after artificial aspiration of colloids but also during the natural course of cystic nodules.

Thyroid nodules which are taller than wide have a 93\% specificity for malignancy [6]. This is thought to be due to a centrifugal tendency in malignant tumors, which does not necessarily occur at a uniform rate in all dimensions [11]. The growth of most benign nodules has been found to remain within the normal tissue plane, whereas malignant nodules grow across the normal tissue plane [12]. In this study, this specific appearance of malignant nodules was less frequently observed in the benign cystic group. It is probable that these benign nodules resulting from the shrinkage of cystic nodules would not grow across the normal tissue plane.

Microcalcification due to calcified psammoma bodies within the nodule is seen in $25 \%-40 \%$ of PTC cases, which is one of the most specific features of thyroid malignancies [11]. In US, microcalcification appears as a punctuate hyperechoic focus without posterior acoustic shadowing [13]. In this study, some PTC patients revealed small sized hyperechogenic dots in US, suggesting microcalcification. There was no significant difference in the prevalence of internal echogenic dots between the 2 groups. However, the benign cystic group had relatively larger echogenic dots compared with the PTC group. The echogenic foci in the benign cystic group were thought to represent debris composed of cellular debris, cholesterol and keratin.

Although the presence of multiple nodules should never be dismissed as a sign of benignity, the probability of malignancy of multiple cystic nodules in US is very low [14]. The multiple thyroid nodules were frequently observed in both cystic benign group and PTC group, whereas other cystic nodules except primary nodule were more frequently found in benign cystic group in this study.

The results of this study are subjected to some limitations. The final diagnosis of cystic nodules in our study was confirmed by cytology with fine needle aspiration, but not by pathology with surgery. A cystic component occurs in some papillary thyroid carcinomas, although cystic carcinomas are rare and account for only 6\% of all malignancies [13]. It was also reported that some cystic carcinomas have a solid component with vascularity, solid excrescences producing into the cyst or microcalcification, which may differentiate a malignant nodule from a benign cystic nodule $[13,15]$. Thus, we excluded predominantly cystic nodules with these US findings. In addition, purely or predominantly cystic appearance included in our study is rarely observed in malignant nodules. However, the pathologic evaluation or further follow-up of these cystic nodules is required for diagnostic accuracy and implication 
of this study. Second, because the echogenicity was evaluated without a numerical and reproductive system such as gray scale, it can be subjective depending on the examiners. Third, echogenic dots observed in the benign cystic group might be difficult to discriminate from microcalcification or macrocalcification in the PTC group and could easily be interpreted as calcification.

In conclusion, some of benign cystic nodules may have suspicious malignant features in US during follow-up period, whether they have previous histories of colloid aspiration or levothyroxine therapy. However, a careful assessment of US finding and previous history may be of value in differentiating between benign cystic nodules with morphological changes mimicking malignancies and small PTC with marked hypoechogenicty.

\section{Disclosure Statement}

All authors of study reported that they have no conflict of interests.

\section{Acknowledgments}

This work was supported by clinical research grant from Pusan National University Hospital (2011).

\section{References}

1. Hodgson NC, Button J, Solorzano CC (2004) Thyroid cancer: is the incidence still increasing? Ann Surg Oncol 11: 1093-1097.

2. Papini E, Guglielmi R, Bianchini A, Crescenzi A, Taccogna S, Nardi F, Panunzi C, Rinaldi R, Toscano V, Pacella CM (2002) Risk of malignancy in nonpalpable thyroid nodules: predictive value of ultrasound and color-Doppler features. J Clin Endocrinol Metab 87: 19411946.

3. Mandel SJ (2004) Diagnostic use of ultrasonography in patients with nodular thyroid disease. Endocr Pract 10: 246-252.

4. Frates MC, Benson CB, Charboneau JW, Cibas ES, Clark OH, Coleman BG, Cronan JJ, Doubilet PM, Evans DB, Goellner JR, Hay ID, Hertzberg BS, Intenzo CM, Jeffrey RB, Langer JE, Larsen PR, Mandel SJ, Middleton WD, Reading CC, Sherman SI, Tessler FN (2005) Management of thyroid nodules detected at US: Society of Radiologists in Ultrasound consensus conference statement. Radiology 237: 794-800.

5. Fish SA, Langer JE, Mandel SJ (2008) Sonographic imaging of thyroid nodules and cervical lymph nodes. Endocrinol Metab Clin North Am 37: 401-417.

6. Kim EK, Park CS, Chung WY, Oh KK, Kim DI, Lee JT, Yoo HS (2002) New sonographic criteria for recommending fine-needle aspiration biopsy of nonpalpable solid nodules of the thyroid. Am J Roentgenol 178: 687691.

7. JS Park, DW Kim, CK Eun, SJ Choi, MH Rho (2008) Long-term follow-up sonography of benign cystic thyroid nodules after a percutaneous ethanol injection. $J$ Korean Radiol Soc 58: 21-28.
8. Koo JH, Shin JH, Han BK, Ko EY, Kang SS (2010) Cystic thyroid nodules after aspiration mimicking malignancy: sonographic characteristics. J Ultrasound Med 29:1415-1421.

9. Kuma K, Matsuzuka F, Yokozawa T, Miyauchi A, Sugawara M (1994) Fate of untreated benign thyroid nodules: results of long-term follow-up. World J Surg 18: 495-498.

10. Peccin S, de Castsro JA, Furlanetto TW, Furtado AP, Brasil BA, Czepielewski MA (2002) Ultrasonography: is it useful in the diagnosis of cancer in thyroid nodules? $J$ Endocrinol Invest 25: 39-43.

11. Hoang JK, Lee WK, Lee M, Johnson D, Farrell S (2007) US Features of thyroid malignancy: pearls and pitfalls. Radiographics 27: 847-860.

12. Stavros AT, Thickman D, Rapp CL, Dennis MA, Parker SH, Sisney GA (1995) Solid breast nodules: use of sonography to distinguish between benign and malignant lesions. Radiology 196: 123-134.

13. Chan BK, Desser TS, McDougall IR, Weigel RJ, Jeffrey RB Jr (2003) Common and uncommon sonographic features of papillary thyroid carcinoma. $J$ Ultrasound Med 22: 1083-1090.

14. Horvath E, Majlis S, Rossi R, Franco C, Niedmann JP, Castro A, Dominguez M (2009) An ultrasonogram reporting system for thyroid nodules stratifying cancer risk for clinical management. J Clin Endocrinol Metab 94: 1748-1751.

15. Hatabu H, Kasagi K, Yamamoto K, Iida Y, Misaki T, Hidaka A, Shibata T, Shibata T, Shoji K, Higuchi K (1991) Cystic papillary carcinoma of the thyroid gland: a new sonographic sign. Clin Radiol 43: 121-124. 